MATHEMATICS OF COMPUTATION

Volume 67, Number 222, April 1998, Pages 821-831

S $0025-5718(98) 00923-5$

\title{
DIAMETERS OF COMPLETE SETS OF CONJUGATE ALGEBRAIC INTEGERS OF SMALL DEGREE
}

\author{
MICHEL GRANDCOLAS
}

\begin{abstract}
We give bounds for the coefficients of a polynomial as functions of the diameter of its roots, hence we obtain polynomials with minimal diameters and small degree
\end{abstract}

\section{INTRODUCTION}

Let $P$ be a monic irreducible polynomial with coefficients in $\mathbb{Z}$. The set of the roots of $P$ in $\mathbb{C}$ is called a "complete set of conjugate algebraic integers". For example, each point of $\mathbb{Z}$ constitutes such a set. Later, we will ignore this trivial case and we will not distinguish between two complete sets of conjugate algebraic numbers if we can deduce one from the other by an integral translation (i.e. $z \rightarrow z+h$ with $h$ integer) or by a symmetry with respect to the origin (i.e. $z \rightarrow-z)$.

We know [1] that the diameter of such a set, which we also call the diameter of $P$, is at least $\sqrt{3}$ and that, for each real number $c<2$, the number of sets whose diameter is at most $\mathrm{c}$ is finite [2]. These references solve problems studied by Favard [3] in 1920. Robinson [4] found real sets with diameters smaller than 4 and a cardinality smaller than 8 , and has given a list for 7 and 8 , without proving its completeness. Next, at the beginning of 1980, C. W. Lloyd-Smith [5] found these sets with diameters $<2$ and a cardinality $\leq 5$.

The aim of this paper is to generalize these results up to degree 10, by an efficient algorithm. The bounds for the coefficients will be easy to compute for all degrees from the barycenter of the roots and the center of a hexagon containing the roots. Furthermore they will be nearly optimal.

Plan of the proof: In section 3 we deduce an upper bound for the value of $|P(z)|$ from a geometric argument given in [1]. In section 4, using Parseval's identity and Cauchy's formula we give upper bounds for the coefficients of $P$. In section 5 we give nearly optimal bounds in particular cases obtained with Newton's formulae. In section 6 we describe the algorithm we use. It is based on the Gauss-Lucas theorem and formulae of sections 4 and 5 . In section 2 we give the list of all polynomials which are monic, irreducible of degree inferior to 10 and which have a diameter inferior to 2 (or which have the smallest diameters).

I am indebted to M. Langevin for suggesting the study of this problem and to G. Rhin for interesting discussions.

Received by the editor March 10, 1996 and, in revised form, October 25, 1996.

1991 Mathematics Subject Classification. Primary 11Y40, 11R09.

Key words and phrases. Polynomial, diameter, conjugate algebraic integers.

(C)1998 American Mathematical Society 


\section{REsults}

2.1. Main Results. The polynomials $P_{d}(z)$ represent monic irreducible polynomials with coefficients in $\mathbb{Z}$, of degree $d$ and the coefficient of $z^{d-1}$ is an integer between $\left\lceil\frac{-d}{2}\right\rceil$ (where $\lceil x\rceil$ denotes the smallest integer greater than or equal to $x$ ) and 0 , the polynomials $P_{d}(z)$ of minimal diameters are the following:

$$
\begin{gathered}
d=2: z^{2}-z+1, \text { diam }=\sqrt{3}, \text { disc }=-3 \\
d=3: z^{3}-z^{2}+1, \text { diam }=1.79423415 \ldots, \text { disc }=-23 \\
d=4: z^{4}-2 z^{3}+2 z^{2}-z+1, \text { diam }=1.89882892 \ldots, \text { disc }=117 \\
d=5: z^{5}-2 z^{4}+z^{3}+z^{2}-z-1, \text { diam }=1.99179625 \ldots, \text { disc }=3089 \\
d=6: z^{6}-z^{5}+z^{4}-z^{3}+z^{2}-z+1, \text { diam }=1.94985582 \ldots, \text { disc }=-16807 \\
d=7: z^{7}-2 z^{6}+2 z^{5}-2 z^{4}+z^{3}+1, \text { diam }=1.97030662 \ldots, \text { disc }=-438599 \\
d=8: z^{8}-z^{7}+z^{5}-z^{4}+z^{3}-z+1, \text { diam }=1.98904379 \ldots, \text { disc }=1265625 \\
d=9: z^{9}-3 z^{8}+4 z^{7}-3 z^{6}+z^{5}+1, \text { diam }=2.00758717 \ldots, \text { disc }=327060313 \\
d=10: z^{10}-z^{9}+z^{8}-z^{7}+z^{6}-z^{5}+z^{4}-z^{3}+z^{2}-z+1, \text { diam }=1.97964288 \ldots \\
\text { disc }=-2357947691
\end{gathered}
$$

where diam denotes the diameter of the set of roots of the polynomial $P_{d}$ and disc its discriminant.

Remarks. 1) $d=9$ is the only case with diam $>2$. We can conjecture that the only polynomials of diameter less than 2 are cyclotomic for $d \geq 9$. The polynomials of minimal diameter are precisely those which have the smaller discriminants for $d=2,3,4$. This method can give small discriminants.

2) for $d=10$ the search is not exhaustive.

2.2. Other Results. We give the list of polynomials of diameter $<2$ from degree 2 to degree 10 , if the barycenter is in the interval $[0,1 / 2]$ :

degree 2:

$$
\begin{aligned}
& z^{2}-z+1, \text { diam }=\sqrt{3} \\
& \text { degree } 3: \\
& z^{3}-z^{2}+1, \text { diam }=1.794234155701 \\
& z^{3}-z^{2}-1, \text { diam }=1.874181059385 \\
& \text { degree } 4: \\
& z^{4}-2 z^{3}+2 z^{2}-z+1, \text { diam }=1.898828922115 \\
& z^{4}-z^{3}+z^{2}-z+1, \text { diam }=1.902113032590 \\
& z^{4}-z^{3}+1, \text { diam }=1.993919104324 \\
& z^{4}-z+1, \text { diam }=1.993919104324 \\
& \text { degree } 5: \\
& z^{5}-2 z^{4}+z^{3}+z^{2}-z-1, \text { diam }=1.991796253434
\end{aligned}
$$




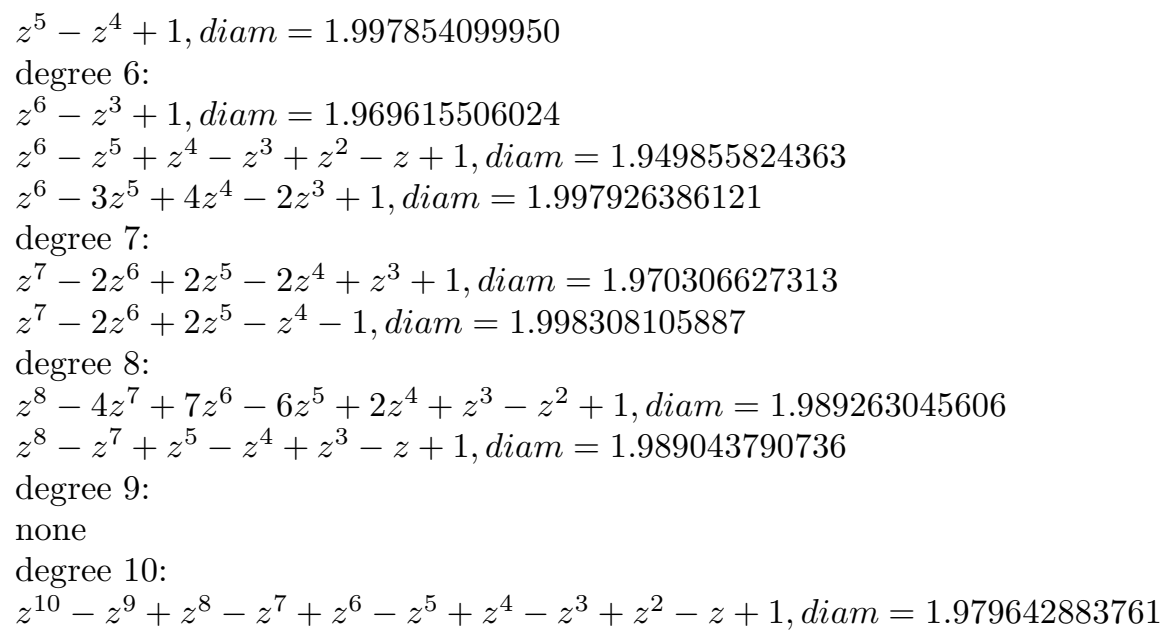

\section{THE GEOMETRICAL LEMMAS}

3.1. Lemma 1. Let $X$ be a subset of diameter $\delta$ symmetric with respect to the real axis. There exists a regular hexagon which contains $X$ and such that the opposite sides are parallel and the distance between these sides is $\delta$.

Proof. According to the argument used in [1], each hexagon formed by the intersection of 3 strips of width $\delta$ satisfies the conditions of the lemma, the first strip being parallel and symmetric with respect to the real axis, the second of the same width making an angle of $\frac{\pi}{3}$ with the real axis, the third being the reflection of the second in the real axis. In particular, $X$ is contained in a disc of radius $\frac{\delta}{\sqrt{3}}$.

3.2. Corollary. If the diameter of the roots of $P$ is $\delta$ and their barycenter is 0 , then the real parts of these roots lie in the segment $[-\alpha+h, \alpha+h]$ with $\alpha=\frac{\delta}{\sqrt{3}}$ and $0 \leq h \leq \frac{\delta}{\sqrt{3}}$.

Proof. By a translation, we can put the barycenter of the roots at the origin. Thanks to a symmetry with respect to the origin, it is always possible to have the center $h$ of the regular hexagon of Lemma 1 in the segment $\left[0, \frac{\delta}{\sqrt{3}}\right]$.

3.3. Lemma 2. Let $M_{i}(1 \leq i \leq d)$ be $d$ points of the complex plane and $G$ their barycenter. Then, for any point $M$,

$$
\frac{\left(\sum_{i=1}^{d} M M_{i}^{2}\right)}{d} \leq M G^{2}+\frac{\delta^{2}}{3} .
$$

Proof. From the equation

$$
\sum_{i=1}^{d} M M_{i}^{2}=d \times M G^{2}+\sum_{i=1}^{d} G M_{i}^{2} \quad \text { and } \quad \sum_{i=1}^{d} J M_{i}^{2}=d \times J G^{2}+\sum_{i=1}^{d} G M_{i}^{2}
$$

(where $J$ is the center of the regular hexagon described in Lemma 1), we deduce the result by noticing that $J M_{i}^{2} \leq \frac{\delta^{2}}{3}$. 
3.4. Corollary. Let $P$ be a polynomial with real coefficients, of degree $d$, where the coefficient of $z^{d}$ (resp. $z^{d-1}$ ) is $a_{d}$ (resp.- $a_{d} d g$, where $g$ is the barycenter of the roots of $P$ ). Then, for all complex numbers $z$,

$$
|P(z)|^{\frac{2}{d}} \leq\left|a_{d}\right|^{\frac{2}{d}}\left(\frac{\delta^{2}}{3}+|z-g|^{2}\right) .
$$

Proof. Apply the inequality of the arithmetic and geometric means to (3.1).

3.5. Lemma 3. Let $\alpha>0, h$ be two reals and $d$ an integer greater than or equal to 2. For $x_{1}, x_{2}, \ldots, x_{d}$, d real, such that

$$
-\alpha+h \leq x_{i} \leq \alpha+h, \sum_{i=1}^{d} x_{i}=0,
$$

we define

$$
\begin{aligned}
& \sigma_{1}(h)=\sum_{i=1, x_{i}>0}^{d} x_{i}=-\sum_{i=1, x_{i}<0}^{d} x_{i}=\frac{1}{2} \sum_{i=1}^{d}\left|x_{i}\right|, \\
& \sigma_{2}(h)=\sum_{i=1}^{d} x_{i}^{2}, \sigma_{3}(h)=\sum_{i=1}^{d} x_{i}^{3}, \sigma_{4}(h)=\sum_{i=1}^{d} x_{i}^{4} .
\end{aligned}
$$

We have:

1) If either $\sigma_{1}(h), \sigma_{2}(h)$ or $\sigma_{4}(h)$ take a maximal value, then we can suppose that, with at most one exception, the values of $x_{i}$ are all equal to $-\alpha+h$ or $\alpha+h$.

2) If $\sigma_{3}(h)$ is positive and maximal, then we can have exactly two possibilities: (i) with at most one exception, the values of $x_{i}$ are all equal to $-\alpha+h$ or $\alpha+h$, or else (ii) several of the $x_{i}$ are negative and equal and the remaining $x_{i}$ are equal to $\alpha+h$.

3) If $\sigma_{3}(h)$ is negative and has a maximal absolute value, then we can have exactly two possibilities: (i) with at most one exception, the values of $x_{i}$ are all equal to $-\alpha+h$ or $\alpha+h$, or else (ii) several of the $x_{i}$ are positive and equal and the remaining $x_{i}$ are equal to $-\alpha+h$.

Remark. this lemma allows us to transform the search for maximal bounds of all the sets of conjugate algebraic integers to a finite number of them. In section 5 , we apply this lemma to $\alpha$ and $h$ found in Lemma 1 of section 3.1 and corollary of section 3.2. The barycenter of the roots $x_{i}$ is at the origin and clearly in $[-\alpha+h, \alpha+h]$.

Proof. 1) case of $\sigma_{1}(h)$ :

(a) Suppose that we have two indices $i \neq j$ such that $-\alpha+h<x_{i} \leq 0 \leq x_{j}<$ $\alpha+h$ and $\sigma_{1}(h)$ has a local maximum. Then we have a real $t>0$ such that:

$-\alpha+h<x_{i}-t<0<x_{j}+t<\alpha+h$ and: $\left|x_{j}+t\right|+\left|x_{i}-t\right|>\left|x_{j}\right|+\left|x_{i}\right|$ which gives a contradiction.

(b) Suppose that we have two indices $i \neq j$ such that $0 \leq x_{i} \leq x_{j}<\alpha+h$ and $\sigma_{1}(h)$ has a local maximum. Then we can choose a real $t>0$ such that: $x_{j}+t=\alpha+h$;

if $x_{i}-t \geq 0$, then $\left|x_{j}+t\right|+\left|x_{i}-t\right|=\left|x_{j}\right|+\left|x_{i}\right|$ hence we can suppose one of the roots is equal to $\alpha+h$.

if $x_{i}-t<0$, then $\left|x_{j}+t\right|+\left|x_{i}-t\right|>\left|x_{j}\right|+\left|x_{i}\right|$ which gives a contradiction.

(c) Suppose that we have two indices $i \neq j$ such that $-\alpha+h<x_{i}<x_{j}<0$ and $\sigma_{1}(h)$ has a local maximum. Then we can choose a real $t>0$ such that: $x_{i}-t=-\alpha+h$;

if $x_{j}+t \leq 0$, then $\left|x_{j}+t\right|+\left|x_{i}-t\right|=\left|x_{j}\right|+\left|x_{i}\right|$ hence we can suppose one of the roots is equal to $-\alpha+h$.

if $x_{j}+t>0$, then $\left|x_{j}+t\right|+\left|x_{i}-t\right|>\left|x_{j}\right|+\left|x_{i}\right|$ which gives a contradiction. 
1) case of $\sigma_{2}(h)$ :

Suppose that we have two indices $i \neq j$ such that $-\alpha+h<x_{i} \leq x_{j}<\alpha+h$ and $\sigma_{2}(h)$ has a local maximum. Then we have a real $t>0$ such that:

$-\alpha+h \leq x_{i}-t<x_{j}+t \leq \alpha+h$ and: $\left(x_{i}-t\right)^{2}+\left(x_{j}+t\right)^{2}-x_{i}^{2}-x_{j}^{2}=$ $2\left(x_{j}-x_{i}\right) t+t^{2}>0$ which gives a contradiction.

1) case of $\sigma_{4}(h)$ :

Suppose that we have two indices $i \neq j$ such that $-\alpha+h<x_{i} \leq x_{j}<\alpha+h$ and $\sigma_{4}(h)$ has a local maximum. Then we have a real $t>0$ such that:

$\left(x_{i}-t\right)^{4}+\left(x_{j}+t\right)^{4}-x_{i}^{4}-x_{j}^{4}=4\left(x_{j}^{3}-x_{i}^{3}\right) t+6\left(x_{j}^{2}+x_{i}^{2}\right) t^{2}+4\left(x_{j}-x_{i}\right) t^{3}+2 t^{4}>0$ which gives a contradiction.

2) Suppose that we have two indices $i \neq j$ such that $-\alpha+h<x_{i} \leq x_{j}<\alpha+h$ and $\sigma_{3}(h)$ has a local maximum in $\left(x_{1}, x_{2}, \ldots, x_{d}\right)$. Since $\left(x_{i}+t\right)^{3}+\left(x_{j}-t\right)^{3}-x_{i}^{3}-x_{j}^{3}=$ $3\left(x_{i}+x_{j}\right) t\left(t+x_{i}-x_{j}\right)$ we have three cases:

(1) first case: $x_{j} \neq x_{i}, x_{j} \neq-x_{i}$.

a) $x_{i}+x_{j}>0$ : we replace $x_{i}$ by $x_{i}+t$ and $x_{j}$ by $x_{j}-t$ with $t<0$ and we have a contradiction.

b) $x_{i}+x_{j}<0$ : we replace $x_{i}$ by $x_{i}+t$ and $x_{j}$ by $x_{j}-t$ with $t>0$ and we have a contradiction.

(2) second case: $x_{j}=-x_{i}$.

We can put one of the roots in $-\alpha+h$ or in $\alpha+h$ without changing the maximum of $\sigma_{3}(h)$.

(3) third case: $x_{j}=x_{i}, x_{j} \neq-x_{i}$.

a) $x_{i}+x_{j}>0:\left(x_{i}+t\right)^{3}+\left(x_{j}-t\right)^{3}-x_{i}^{3}-x_{j}^{3}>0$ and we have a contradiction.

b) $x_{i}+x_{j}<0$ : we cannot have a value $x_{k}<x_{i}$ thanks to the first case. We can suppose there is no root $x_{i}<x_{k}<\alpha+h$ thanks to the first or second case. Hence the other roots are equal to $\alpha+h$ or $x_{i}$. Only in this case we obtain the possibility (ii).

3) The proof is similar to 2).

\section{INEQUALITIES USED IN THE ALGORITHM}

Let $P(z)=\sum_{i=0}^{d} a_{i} z^{i}$ be a polynomial with real coefficients. By computing the mean value of $|P(z)|^{2}$ on a circle of radius $r>0$ centered at the origin, we deduce from (3.2) and Parseval's identity that

$$
\sum_{i=0}^{d}\left|a_{i}\right|^{2} r^{2 i} \leq\left|a_{d}\right|^{2} \int_{0}^{1}\left(\frac{\delta^{2}}{3}+r^{2}-2 g r \cos (2 \pi t)+g^{2}\right)^{d} d t
$$

with $\mathrm{g}=\frac{-a_{d-1}}{d a_{d}}$.

We deduce also from (3.2) and from Cauchy's integral formula an upper bound for the values of the coefficients of $P$ :

$$
\left|a_{i}\right| \leq\left|a_{d}\right| \frac{\int_{0}^{1}\left(\frac{\delta^{2}}{3}+r^{2}-2 g r \cos (2 \pi t)+g^{2}\right)^{\frac{d}{2}} d t}{r^{i}}, \quad 0 \leq i \leq d-1 .
$$

This yields for $g=0$ :

$$
\left|a_{i}\right| \leq\left|a_{d}\right| \frac{\left(r^{2}+\delta^{2} / 3\right)^{d / 2}}{r^{i}}, \quad 0 \leq i \leq d-1 .
$$

We will look for numerical values of $r$ minimizing these bounds.

We still assume that the polynomial $P$ is monic, that is to say: $P(z)=z^{d}+$ $b_{1} z^{d-1}+\cdots+b_{k} z^{d-k}+\cdots+b_{d}$. We will suppose also that it is of diameter $<2$ 
for the computation of minimal diameters and that the barycenter of its roots is in the interval $[0,1 / 2]$ (this assumption is not restrictive because we can replace $P(z)$ by $P( \pm z+h), h \in \mathbb{Z})$. This limits the possible values of $b_{1}$.

We reduce the computations significantly by applying the above bounds successively to the derivatives of $P$. This is possible because the sets of corresponding roots of these derivations lie in the same hexagon as we have seen earlier, by the theorem of Gauss-Lucas. These sets also have the same barycenter $g$. Hence we replace the bounds of a quadratic form in $n$ variables by a sequence of triangular quadratic forms. The preceding remark shows that we can consider a sequence of quadratic forms with the variables $\left(b_{1}, \ldots, b_{k}\right)$ for $1 \leq k \leq d$.

It can also be useful, when the degree increases, to compute with rational coefficients and put the barycenter of the roots at the origin. Indeed, if we consider $P(z)=z^{d}+b_{1} z^{d-1}+\cdots+b_{k} z^{d-k}+\cdots+b_{d}$, the coefficients of the polynomial $Q(x)=x^{d}+B_{1} x^{d-1}+\cdots+B_{k} x^{d-k}+\cdots+B_{d}$, obtained from $P$ by a translation of the barycenter of the roots to the origin, satisfies the system of formulae:

$$
\begin{gathered}
B_{1}=0 \\
B_{2}=\left(\begin{array}{c}
d \\
d-2
\end{array}\right)\left(\frac{-b_{1}}{d}\right)^{2}+b_{1}\left(\begin{array}{l}
d-1 \\
d-2
\end{array}\right)\left(\frac{-b_{1}}{d}\right)+b_{2} \\
\ldots \\
B_{k}=\left(\begin{array}{c}
d \\
d-k
\end{array}\right)\left(\frac{-b_{1}}{d}\right)^{k}+b_{1}\left(\begin{array}{l}
d-1 \\
d-k
\end{array}\right)\left(\frac{-b_{1}}{d}\right)^{k-1}+\ldots+b_{j}\left(\begin{array}{c}
d-j \\
d-k
\end{array}\right)\left(\frac{-b_{1}}{d}\right)^{k-j}+\cdots+b_{k} \\
\ldots \\
B_{d}=\left(\frac{-b_{1}}{d}\right)^{d}+b_{1}\left(\frac{-b_{1}}{d}\right)^{d-1}+\cdots+b_{j}\left(\frac{-b_{1}}{d}\right)^{k-j}+\cdots+b_{d}
\end{gathered}
$$

The interest of replacing coefficient $b_{i}$ with $B_{i}$ is that the number of possible values for $B_{i}$ is smaller because the bounds previously obtained are increasing functions of the barycenter.

\section{Bounds With Newton's formulae}

We suppose in this section that the barycenter of the roots $z_{i}$ of $P$ is at the origin, furthermore $h$ : the center of the hexagon containing the roots is positive by Lemma 1 of section 3 . We can find nearly optimal bounds for $B_{2}, B_{3}, B_{4}$ by using Newton's formulae and Lemma 3 of section 3. Indeed we apply the results of Lemma 3 to the real parts of the roots $z_{i}$ which lie in the segment $[-\alpha+h, \alpha+h]$ by Corollary 3.2.

5.1. Lemma. For a polynomial $P$, we introduce the following expressions, where $z_{j}=x_{j}+i y_{j}, 1 \leq j \leq d$, are its roots :

$$
\begin{gathered}
\sigma_{1}(h)=\sum_{j=1, x_{j}>0}^{d} x_{j}, \\
\sigma_{2}(h)=\sum_{j=1}^{d} x_{j}^{2}, \sigma_{3}(h)=\sum_{j=1}^{d} x_{j}^{3}, \sigma_{4}(h)=\sum_{i=1}^{d} x_{j}^{4} .
\end{gathered}
$$


The bounds of $\sigma_{1}(h), \sigma_{2}(h),\left|\sigma_{3}(h)\right|, \sigma_{4}(h)$ are found thanks to Lemma 3 and given by:

1) For $\sigma_{1}(h), \sigma_{2}(h), \sigma_{4}(h)$ we are in the first case of Lemma 3, h,k satisfy the following conditions:

$0 \leq \frac{[-2 \alpha k+\alpha(d-2)]}{d} \leq h \leq \alpha-\frac{2 \alpha k}{d}$,

$k$ is the number of $x_{i}=\alpha+h, k \in\left[0, \frac{(d-1)}{2}\right]$ if $d$ is odd, $k \in\left[0, \frac{d}{2}-1\right]$ if $d$ is even.

The condition on $h$ is found thanks to the root which is not equal to $-\alpha+h$ or $\alpha+h$.

$$
\begin{aligned}
& 0 \leq \sigma_{1}(h) \leq k(\alpha+h)+|(-d+1) h-2 \alpha k+\alpha(d-1)| \\
& 0 \leq \sigma_{2}(h) \leq k(\alpha+h)^{2}+(d-1-k)(-\alpha+h)^{2} \\
&+ {[(-d+1) h-2 \alpha k+\alpha(d-1)]^{2} } \\
& 0 \leq \sigma_{4}(h) \leq k(\alpha+h)^{4}+(d-1-k)(-\alpha+h)^{4} \\
&+ {[(-d+1) h-2 \alpha k+\alpha(d-1)]^{4} . }
\end{aligned}
$$

2) For $\left|\sigma_{3}(h)\right|$, we have three bounds $B_{k}(h), B_{k^{\prime}}(h), B_{k^{\prime \prime}}(h)$ which correspond respectively to the three cases of Lemma 3:

$$
0 \leq\left|\sigma_{3}(h)\right| \leq \max _{k, k^{\prime}, k^{\prime \prime}}\left\{B_{k}(h), B_{k^{\prime}}(h), B_{k^{\prime \prime}}(h)\right\}
$$

with:

a) $B_{k}(h)=\left|k(\alpha+h)^{3}+(d-1-k)(-\alpha+h)^{3}+[(-d+1) h-2 \alpha k+\alpha(d-1)]^{3}\right|$, $h, k$ satisfy the following conditions:

$0 \leq \frac{[-2 \alpha k+\alpha(d-2)]}{d} \leq h \leq \alpha-\frac{2 \alpha k}{d}$,

$k$ is the number of $x_{i}=\alpha+h, k \in\left[0, \frac{d-1}{2}\right]$, if $d$ is odd, $k \in\left[0, \frac{d}{2}-1\right]$ if $d$ is even.

b) $\left.B_{k^{\prime}}(h)=\mid k^{\prime}(\alpha+h)^{3}+\left[-k^{\prime}(\alpha+h)\right]^{3} /\left(d-k^{\prime}\right)^{2}\right\} \mid$.

$h, k$ ' satisfy the following conditions:

$0 \leq h \leq \alpha\left(1-\frac{2 k^{\prime}}{d}\right), k^{\prime}$ is the number of $x_{i}=\alpha+h, d-k^{\prime}$ is the number of negative roots which are equal, $k^{\prime} \in\left[0, \frac{d-1}{2}\right]$ if $d$ is odd, $k^{\prime} \in\left[0, \frac{d}{2}-1\right]$ if $d$ is even.

c) $B_{k^{\prime \prime}}(h)=\left|k^{\prime \prime}(-\alpha+h)^{3}+\left[k^{\prime \prime}(\alpha-h)\right]^{3} /\left(d-k^{\prime \prime}\right)^{2}\right|$.

$h, k^{\prime \prime}$ satisfy the following conditions:

$\max \left(0, \alpha\left(\frac{2 k^{\prime \prime}}{d}-1\right)\right) \leq h \leq \alpha, k^{\prime \prime}$ is the number of $x_{i}=-\alpha+h, d-k^{\prime \prime}$ is the number of positive roots which are equal, $k^{\prime \prime} \in[1, d-1]$.

Remarks. 1) It would be possible to find bounds using the sign of $\sigma_{3}(h)$, but it is not useful.

2) We can observe that:

$\max _{h} \max _{k, k^{\prime}, k^{\prime \prime}}\left\{B_{k}(h), B_{k^{\prime}}(h), B_{k^{\prime \prime}}(h)\right\}=\max _{h} \max _{k} B_{k}(h)$.

5.2. Corollary 1. For each function $\sigma_{i}(h)$, one uses calculus to maximize the right member of each inequality as a function of $h$ for fixed $k$ and then maximizes over the various possibilities for $k$. We call $\sigma_{1}, \sigma_{2}, \sigma_{3}, \sigma_{4}$ these bounds. The maximum of $\sigma_{i}(h),(2 \leq i \leq 4)$, is obtained with an algorithm thanks to the derivatives below and the intervals obtained in section 5.1.

Derivatives:

We give here the derivatives to find $\sigma_{2}, \sigma_{3}, \sigma_{4}$ obtained with the PARI system. 1) derivative of $f$,

$$
f(h)=k(\alpha+h)^{2}+(d-1-k)(-\alpha+h)^{2}+[(-d+1) h-2 \alpha k+\alpha(d-1)]^{2}:
$$




$$
f^{\prime}(h)=\left(2 d^{2}-2 d\right) h-2 \alpha d^{2}+2 d \alpha+4 d \alpha k .
$$

2) derivative of $g$,

$$
\begin{aligned}
g(h)= & k(\alpha+h)^{3}+(d-k-1)(-\alpha+h)^{3}+[(-d+1) h-2 \alpha k+\alpha(d-1)]^{3}: \\
g^{\prime}(h)= & \left(-3 d^{3}+9 d^{2}-6 d\right) h^{2}+\left[\left(-12 d^{2}+24 d\right) \alpha k+\left(6 d^{3}-18 d^{2}+12 d\right) \alpha\right] h \\
& +(-12 d+12) \alpha^{2} k^{2}+\left(12 d^{2}-24 d+12\right) \alpha^{2} k+\left(-3 d^{3}+9 d^{2}-6 d\right) \alpha^{2} .
\end{aligned}
$$

3) derivative of $l, l(h)=k(\alpha+h)^{3}+\frac{[-k(\alpha+h)]^{3}}{(d-k)^{2}}$ :

$l^{\prime}(h)=\left[\frac{\left(-6 d k^{2}+3 d^{2} k\right)}{\left(k^{2}-2 d k+d^{2}\right)}\right] h^{2}+\left[\frac{\left(-12 d \alpha k^{2}+6 d^{2} \alpha k\right)}{\left(k^{2}-2 d k+d^{2}\right)}\right] h+\left[\frac{\left(-6 d \alpha^{2} k^{2}+3 d^{2} \alpha^{2} k\right)}{\left(k^{2}-2 d k+d^{2}\right)}\right]$.

4) derivative of $m$,

$$
\begin{aligned}
m(h)=k( & +h)^{4}+(d-k-1)(-\alpha+h)^{4}+[(-d+1) h-2 \alpha k+\alpha(d-1)]^{4}: \\
m^{\prime}(h)= & \left.4 d^{4}-16 d^{3}+24 d^{2}-12 d\right] h^{3} \\
& +\left[\left(24 d^{3}-72 d^{2}+72 d\right) \alpha k+\left(-12 d^{4}+48 d^{3}-72 d^{2}+36 d\right) \alpha\right] h^{2} \\
& +\left[\left(48 d^{2}-96 d+48\right) \alpha^{2} k^{2}+\left(-48 d^{3}+144 d^{2}-144 d+48\right) \alpha^{2} k\right. \\
& \left.+\left(12 d^{4}-48 d^{3}+72 d^{2}-36 d\right) \alpha^{2}\right] h \\
& +\left[(32 d-32) \alpha^{3} k^{3}+\left(-48 d^{2}+96 d-48\right) \alpha^{3} k^{2}\right. \\
& \left.+\left(24 d^{3}-72 d^{2}+72 d-16\right) \alpha^{3} k+\left(-4 d^{4}+16 d^{3}-24 d^{2}+12 d\right) \alpha^{3}\right] .
\end{aligned}
$$

5.3. Corollary 2. Bounds of $B_{2}, B_{3}, B_{4}$ as functions of $\sigma_{1}, \sigma_{2}, \sigma_{3}, \sigma_{4}$ :

$$
\begin{gathered}
\frac{-\sigma_{2}}{2} \leq B_{2} \leq\left\lfloor\frac{d}{2}\right\rfloor\left(\frac{\delta}{2}\right)^{2}, \\
\left|B_{3}\right| \leq \frac{\sigma_{3}}{3}+\sigma_{1}\left(\frac{\delta}{2}\right)^{2}, \\
\left(-\sigma_{4}-d\left(\frac{\delta}{2}\right)^{2}\right) / 4 \leq B_{4} \leq \sigma_{2}\left(\frac{\delta}{2}\right)^{2}+\frac{\sigma_{2}^{2}}{8} .
\end{gathered}
$$

Remark. $B_{1}=0$.

Proof. For (5.5.a) we use $S_{2}=\sum_{i=1}^{d} z_{i}^{2}$, for (5.6.a) $S_{3}=\sum_{i=1}^{d} z_{i}^{3}$, for (5.7.a) $S_{4}=\sum_{i=1}^{d} z_{i}^{4}$.

Since we have: $S_{2}=\sum_{i=1}^{d} x_{i}^{2}-\sum_{i=1}^{d} y_{i}^{2}, S_{3}=\sum_{i=1}^{d} x_{i}^{3}-3 \sum_{i=1}^{d} x_{i} y_{i}^{2}, S_{4}=$ $\sum_{i=1}^{d} x_{i}^{4}-6 \sum_{i=1}^{d} x_{i}^{2} y_{i}^{2}+\sum_{i=1}^{d} y_{i}^{4}$, hence we obtain bounds for $S_{2}, S_{3}, S_{4}$, and thanks to Newton's formulae, $B_{2}=\frac{-S_{2}}{2}, B_{3}=\frac{S_{3}}{3}, B_{4}=\frac{S_{2}^{2}}{8}-\frac{S_{4}}{4}$.

We get the bounds from the following inequalities:

$$
\begin{gathered}
-2\left\lfloor\frac{d}{2}\right\rfloor\left(\frac{\delta}{2}\right)^{2} \leq S_{2} \leq \sigma_{2}, \\
\left|S_{3}\right| \leq \sigma_{3}+3 \sigma_{1}\left(\frac{\delta}{2}\right)^{2}, \\
-4 \sigma_{2}\left(\frac{\delta}{2}\right)^{2} \leq S_{4} \leq \sigma_{4}+d\left(\frac{\delta}{2}\right)^{4},
\end{gathered}
$$

which are obtained by simple considerations on the sign of values $x_{i}$. 
5.4. Corollary 3. If $P$ is totally real, we have:

$$
\begin{gathered}
\frac{-\sigma_{2}}{2} \leq B_{2} \leq 0, \\
\left|B_{3}\right| \leq \frac{\sigma_{3}}{3}, \\
\frac{-\sigma_{4}}{4} \leq B_{4} \leq \frac{\sigma_{2}^{2}}{8} .
\end{gathered}
$$

Remark. If $P$ is totally positive (i.e. all its roots are real and positive), we have:

$$
0 \leq B_{3} \leq \frac{\sigma_{3}}{3}
$$

instead of (5.6.a) because $B_{3}$ cannot be negative.

5.5. Remark. We can find formulae for bounds of $B_{k^{\prime}}(h), B_{k^{\prime \prime}}(h)$; this is a way to verify the bounds of $\sigma_{3}$ found thanks to an algorithm with the formulae of (5.3).

1) For $B_{k^{\prime}}(h)$ :

We can simplify $B_{k^{\prime}}(h)$, so that:

$B_{k^{\prime}}(h)=\left|\frac{k^{\prime}(\alpha+h)^{3} d\left(d-2 k^{\prime}\right)}{\left(d-k^{\prime}\right)^{2}}\right|$ and we get $\max _{k^{\prime} \in K} k^{\prime}\left(d-k^{\prime}\right)\left(d-2 k^{\prime}\right)$ for $k^{\prime}=$ $\frac{d}{2}-\frac{\sqrt{3} d}{6}$; hence: $\max _{h \in I} \max _{k^{\prime} \in K} B_{k^{\prime}}(h)=\max _{k^{\prime} \in K} \frac{8 \alpha^{3} k^{\prime}\left(d-k^{\prime}\right)\left(d-2 k^{\prime}\right)}{d^{2}} \leq \frac{4}{27} d \delta^{3}$.

2) For $B_{k^{\prime \prime}}(h)$ :

We can simplify $B_{k^{\prime \prime}}(h)$, so that:

$B_{k^{\prime \prime}}(h)=\left|\frac{k^{\prime \prime}(-\alpha+h)^{3} d\left(d-2 k^{\prime \prime}\right)}{\left(d-k^{\prime \prime}\right)^{2}}\right|$.

We have two cases:

1) first case: $k^{\prime \prime} \in K=[d / 2, d-1], h \in J=\left[\alpha\left(\frac{2 k^{\prime \prime}}{d}-1\right), \alpha\right]$, $\max _{h \in J} \max _{k^{\prime \prime} \in K} B_{k^{\prime \prime}}(h)=\max _{k^{\prime \prime} \in K} \frac{8 \alpha^{3}\left(d-k^{\prime \prime}\right) k^{\prime \prime}\left(2 k^{\prime \prime}-d\right)}{d^{2}}$.

We get $\max _{k^{\prime \prime} \in K}\left(d-k^{\prime \prime}\right) k^{\prime \prime}\left(2 k^{\prime \prime}-d\right)$ for $k^{\prime \prime}=\frac{d}{2}+\frac{\sqrt{3} d}{6}$; hence: $\max _{h \in J} \max _{k^{\prime \prime} \in K} B_{k^{\prime \prime}}(h) \leq \frac{4}{27} d \delta^{3}$.

2) second case: $k^{\prime \prime} \in K^{\prime}=[1, d / 2], h \in J^{\prime}=[0, \alpha]$,

$\max _{h \in J^{\prime}} \max _{k^{\prime \prime} \in K^{\prime}} B_{k^{\prime \prime}}(h)=\max _{k^{\prime \prime} \in K^{\prime}} \frac{\alpha^{3} k^{\prime \prime} d\left(d-2 k^{\prime \prime}\right)}{\left(d-k^{\prime \prime}\right)^{2}}$.

We get $\max _{k^{\prime \prime} \in K^{\prime}} \frac{k^{\prime \prime}\left(d-2 k^{\prime \prime}\right)}{\left(d-k^{\prime \prime}\right)^{2}}$ for $k^{\prime \prime}=\frac{d}{3}$;

hence: $\max _{h \in J^{\prime}} \max _{k^{\prime \prime} \in K^{\prime}} B_{k^{\prime \prime}}(h) \leq \frac{d}{12 \sqrt{3}} \delta^{3}$.

Thanks to case 1 and 2: $\max _{h \in I} \max _{k^{\prime \prime} \in[1, d-1]} B_{k^{\prime \prime}}(h) \leq \frac{4}{27} d \delta^{3}$.

\section{Plan of the Algorithm}

Let $P_{k}=\frac{(n-k) !}{n !} P^{(k)} . P_{k}$ is a monic polynomial in $\mathbb{Q}[\mathbb{Z}]$ which has a set of roots with diameters smaller than $\delta$ by the theorem of Gauss-Lucas. Now $P_{k}$ depends only on $b_{1}, \ldots, b_{k}$, which allows us to use a recursive algorithm. This method has already been used by Robinson for the search of polynomials with all real roots and a small diameter. We will test from $k=2$ to $k=n$ the polynomials $P_{k}$ which have their diameter less than $\delta$. Hence we have a tree search which allows us to considerably limit the number of polynomials to study, compared to an iterative search on the set of values permitted by the bounds obtained in section 4 . Of course, we must also use Parseval's formula. We need not compute the whole set of roots; we can stop the computation of roots of the polynomial $P_{k}$ if the imaginary part of a root is greater than $\frac{\delta}{2}$ or if we find two roots $x_{i}$ and $x_{j}$ of $P_{k}$ such that $\left|x_{i}-x_{j}\right| \geq \delta$. 
At the beginning, we compute the bounds of $B_{k}$, that is to say: $-B s u p_{k}$ and $B$ sup $_{k}$ and also $h_{r, k}$ bounding $r^{2 k}+\sum_{i=1}^{k} D_{i}^{2} r^{2(k-i)}$ (for a few judicious values of $r$ ) thanks to the formula given in section 4 . Here $D_{i}$ is the coefficient of $x^{k-i}$ of $P_{k}$ translated to the origin. We compute also the values binf $f_{1}=\left\lceil\frac{-n}{2}\right\rceil$ and bsup $_{1}=0$ bounding $b_{1}$.

We construct a procedure test $k_{k}$ which computes the diameter of the roots of $P_{k}$, checks whether it is smaller than $\delta$ and which also verifies the Parseval constraint.

step 1: we iterate the possible values of $b_{1}$

step 2: we iterate the possible values of $b_{2}$, modulo the change of variables of section 4 . If $t_{e s t}$ is satisfied, we go to step 3 , else we go to the next value of $b_{2}$ or we come back to step 1 at the end of the iteration.

step $k$ : we iterate the possible values of $b_{k}$, modulo the change of variables of section 4. If $t_{e s t}$ is satisfied and $k<n$ we go to step $k+1$, if test $t_{k}$ is satisfied and $k=n$ we give the result, else we go to the next value of $b_{k}$ or we come back to step $k-1$ at the end of the iteration.

\section{TABLE OF THE BOUNDS FOR COEFFICIENTS}

1) The bounds of $B_{2}, B_{3}, B_{4}$ are obtained with the formulae of section 5 .

2) The bounds of $B_{5}, B_{6}, B_{7}, B_{8}, B_{9}$ are obtained with formula (4.3) since the barycenter is supposed to be at the origin. The derivative of $r \rightarrow \frac{\left(r^{2}+\delta^{2} / 3\right)^{d / 2}}{r^{i}}, 1 \leq$ $i \leq d-1$, is easy to compute. The minimum of $r \rightarrow \frac{\left(r^{2}+\delta^{2} / 3\right)^{d / 2}}{r^{i}}$ is obtained for $r=\frac{\delta}{\sqrt{3}} \times \sqrt{\frac{i}{(d-i)}}$. For $i=0$ it is obtained with $r=0$.

3) Using the formulae of section 5 instead of section 4, we considerably shorten the time of computation (it divides by 2 or 3 ).

Bounds for $B_{2}, B_{3}, B_{4}, B_{5}$ as a function of the degree if the diameter is 2 :

\begin{tabular}{|c|cc|cc|cc|cc|}
\hline degree $\backslash$ coef & \multicolumn{2}{|c|}{ B2 } & \multicolumn{2}{c|}{ B3 } & \multicolumn{2}{c|}{ B4 } & \multicolumn{2}{c|}{ B5 } \\
\hline 3 & -1.78 & 1 & -2.46 & 2.46 & \multicolumn{2}{c|}{ none } & \multicolumn{2}{c|}{ none } \\
\hline 4 & -2.67 & 2 & -3.85 & 3.85 & -3.34 & 8.91 & \multicolumn{2}{c|}{ none } \\
\hline 5 & -3.2 & 2 & -4.76 & 4.76 & -4.21 & 11.52 & -2.06 & 2.06 \\
\hline 6 & -4 & 3 & -5.76 & 5.76 & -4.96 & 16 & -7.94 & 7.94 \\
\hline 7 & -4.58 & 3 & -6.48 & 6.48 & -5.69 & 19.62 & -16.67 & 16.67 \\
\hline 8 & -5.34 & 4 & -7.70 & 7.70 & -6.67 & 24.91 & -28.95 & 28.95 \\
\hline 9 & -5.93 & 4 & -8.69 & 8.69 & -7.58 & 29.45 & -45.28 & 45.28 \\
\hline
\end{tabular}

Bounds for $B_{6}, B_{7}, B_{8}, B_{9}$ as a function of the degree if the diameter is 2 :

\begin{tabular}{|c|c|c|c|c|}
\hline degree \coef & $\mathrm{B} 6$ & B7 & B8 & B9 \\
\hline 6 & -2.38 & none & none & none \\
\hline 7 & -15.9 & $\begin{array}{ll}-2.74 & 2.74\end{array}$ & none & none \\
\hline 8 & $\begin{array}{ll}-22.48 & 22.48\end{array}$ & $-12.36 \quad 12.36$ & $\begin{array}{ll}-3.17 & 3.17\end{array}$ & none \\
\hline 9 & $\begin{array}{ll}-41.61 & 41.61\end{array}$ & $-29.72 \quad 29.72$ & $-15.20 \quad 15.20$ & $-3.65 \quad 3.65$ \\
\hline
\end{tabular}




\section{REFERENCES}

[1] M. Langevin, E. Reyssat, and G. Rhin: Diamètres transfinis et problème de Favard (Ann. Inst. Fourier Grenoble, 38, 1, (1988), 1-16). MR 90b:11103

[2] M. Langevin: Solutions des problèmes de Favard (Ann. Inst. Fourier Grenoble, 38, 2, (1988), 1-10). MR 90b:11104

[3] J. Favard: Sur les nombres algébriques (C.R.Acad.Sc. Paris, 186, 1928, 1181-1182).

[4] R.M. Robinson: Algebraic equations with span less than 4 (Math. Comp 18 (1964), 547-559). MR 29:6624

[5] C. W. Lloyd-Smith: PhD Thesis Adelaide 1980, Problems on the distribution of conjugates of algebraic numbers.

UfR Mim, Département de Mathématiques, URA CNRS 399, Université de Metz, Ile du Saulcy, 57045 Metz Cedex 01, France

E-mail address: Grandcol@poncelet.univ-metz.fr 